\title{
Acral Melanomas of the Sole May Have Worse Prognosis Compared with Other Sites of Acral Melanoma
}

\author{
Georgia M. Beasley, MD MHSc \\ Duke University, Durham, NC
}

Acral melanoma occurs on skin with much less direct sun exposure-the soles, palms, and nail beds-compared with the skin of sun-exposed regions where other subtypes of cutaneous melanoma occur: the head, neck, truck, and extremities. Therefore, we might expect acral melanomas to have a distinct genesis and biology that may not be as directly linked to sun damage. Indeed, while ultravioletinduced oncogenesis is predominant in cutaneous melanoma, transcriptomic and genomic analyses of acral melanoma have in fact revealed an absence of ultravioletderived tumorigenesis.' Even though the molecular profile is unique, acral melanomas are currently assigned to the same stage categories and treated similarly to other subtypes of cutaneous melanomas. In this issue of Annals of Surgical Oncology, Wei et al. ${ }^{2}$ examine and compare the clinicopathological and survival profiles across primary sites in acral melanoma. These authors describe a series of over 1000 patients with acral melanoma, one of the largest series known to date. Their overall findings can help inform clinicians who take care of patients with this disease about incidence and prognosis related to acral melanoma, including prognosis by anatomic site.

For dermatologists and those evaluating abnormal skin lesions, acral melanoma of the palm is much more rare: sole melanoma accounted for the large majority $(68.5 \%)$ of all the cases in the series, while acral melanoma of the nail bed $(23.3 \%)$ and palm (8.2\%) accounted for less than half of all acral melanomas included in the study. The authors' primary goal was to examine whether melanoma-specific

\footnotetext{
(C) Society of Surgical Oncology 2020
}

First Received: 26 March 2020;

Published Online: 19 April 2020

G. M. Beasley, MD MHSc

e-mail: Georgia.beasley@duke.edu survival (MSS) varied by site of acral melanoma, the sites being soles, palms, and nail beds, with no further subset of the nail beds of the toes or fingers. They found the median MSS of sole disease (65.0 months) to be close to half that of nail bed (112.0 months) and palm (not yet reached).

The differences in MSS between sites can be partially explained primarily by the fact that $33.2 \%$ of patients with sole melanoma presented with stage III/IV disease, compared with only $16.8 \%$ of palm and $24.1 \%$ of nail bed patients presenting with stage III/IV disease. However, the 65.0 month MSS (which means that, at 5.4 years, $50 \%$ of patients had died) still seems perhaps shorter than expected given that the current 5-year survival rate for patients with stage III melanoma in American Joint Committee on Cancer version 8 is $63.6 \%$. Although certainly outcome in stage III disease is highly variable and survival for the subset of stage IV patients would need to be factored in, only $33.3 \%$ of sole patients presented with stage III/IV disease, so the poor outcome for sole melanoma does not appear to be fully accounted for by stage at presentation.

Inadequate staging may also have accounted for the poor prognosis seen in sole patients. In the sole cohort, $86.8 \%$ of patients had $>1 \mathrm{~mm}$ Breslow depth tumors; current guidelines recommend sentinel lymph node biopsy (SLNB) for tumors $>1 \mathrm{~mm}$ thick or $0.8-1 \mathrm{~mm}$ thick with ulceration. ${ }^{4}$ However, only $26.6 \%$ of patients with sole melanoma had a SLNB. The rate of SLNB positivity was $29.4 \%$ for patients in the cohort with sole melanoma, much higher than the $16 \%$ incidence of sentinel node micrometastases seen in the multicenter selective lymphadenectomy (MSLT-1) trial, which did include acral patients. ${ }^{5}$ So it is certainly possible that many patients with sole melanoma could have been upstaged with SLNB and been assigned stage III at diagnosis, which could explain why sole melanoma patients appeared to have such poor prognosis in this cohort. 
Similarly, at least $83.5 \%$ and $80.2 \%$ of palm and nail bed lesions, respectively, should have been considered for SLNB, but only $17.9 \%$ and $24.4 \%$, respectively, had SLNB. Thus, across all three sites of acral melanoma, rates of understaging were similar. The authors did perform Cox regression in the subgroup who underwent SLNB and still found that MSS was significantly worse for sole melanoma (122.1 months) compared with palm and nail bed (not reached). The 122.1 months figure is certainly more in the range of expected MSS for the whole group of sole acral melanoma compared with the 65.0 months reported in the unadjusted analysis. Finally, although the authors still do not fully account for sole melanoma patients presenting at higher stages (and hence expected worse outcomes), there does seem to be a trend for patients with sole melanoma to do worse compared with nail bed and palm melanoma patients, which can help inform clinicians and patients. Although the study did not make comparisons with other subtypes of cutaneous melanoma, the prognosis for patients with acral melanoma, in particular of the sole, also seems to have a trend toward less favorable prognosis compared with other types of cutaneous melanoma. Overall, most clear is that patients with acral sole melanoma tend to present at higher stages compared with other sites of acral disease; factors driving that could be biologic or patient related.

In one of the largest known series to date, patients with acral melanoma of the sole appear to present at higher stages, including higher rates of SLNB positivity, compared with other subtypes of cutaneous melanoma and other sites of acral melanoma (palm and nail bed). This information can help guide both prognostic and therapeutic implications. Further work should build on the molecular differences that may distinguish acral melanoma from other subtypes of cutaneous melanoma. ${ }^{1}{ }^{6}$ Molecular analysis not only may explain the observed differences in outcomes for patients with acral melanoma compared with other subtypes of cutaneous melanoma but also can prompt development of specific and effective therapies for patients with acral melanoma.

DISCLOSURE Consultant Regeneron 2019

\section{REFERENCES}

1. Liang WS, Hendricks W, Kiefer J, Schmidt J, Sekar S, Carpten J, Craig DW, Adkins J, Cuyugan L, Manojlovic Z, Halperin RF, Helland A, Nasser S, Legendre C, Hurley LH, Sivaprakasam K, Johnson DB, Crandall H, Busam KJ, Zismann V, Deluca V, Lee J, Sekulic A, Ariyan CE, Sosman J, Trent J: Integrated genomic analyses reveal frequent TERT aberrations in acral melanoma. Genome Res 27:524-532, 2017PMC5378171

2. Wei $\mathrm{X}, \mathrm{Wu} \mathrm{D}, \mathrm{Li} \mathrm{H}$, et al. The clinicopathological and survival profiles comparison across primary sites in acral melanoma. Ann Surg Oncol. 2020. https://doi.org/10.1245/s10434-020-08418-5

3. Gershenwald JE, Scolyer RA. Melanoma staging: American Joint Committee on Cancer (AJCC) 8th edition and beyond. Ann Surg Oncol 25:2105-2110, 2018

4. Wong SL, Faries MB, Kennedy EB, Agarwala SS, Akhurst TJ, Ariyan C, Balch CM, Berman BS, Cochran A, Delman KA, Gorman M, Kirkwood JM, Moncrieff MD, Zager JS, Lyman GH: Sentinel lymph node biopsy and management of regional lymph nodes in melanoma: American Society of Clinical Oncology and Society of Surgical Oncology clinical practice guideline update. Ann Surg Oncol 25:356-377, 2018

5. Morton DL, Thompson JF, Cochran AJ, Mozzillo N, Elashoff R, Essner R, Nieweg OE, Roses DF, Hoekstra HJ, Karakousis CP, Reintgen DS, Coventry BJ, Glass EC, Wang HJ: Sentinel-node biopsy or nodal observation in melanoma. N Engl J Med 355:130717,2006

6. Kong Y, Sheng X, Wu X, Yan J, Ma M, Yu J, Si L, Chi Z, Cui C, Dai J, Li Y, Yu H, Xu T, Tang H, Tang B, Mao L, Lian B, Wang $X$, Yan X, Li S, Guo J: Frequent genetic aberrations in the CDK4 pathway in acral melanoma indicate the potential for CDK4/6 inhibitors in targeted therapy. Clin Cancer Res 23:6946-6957, 2017

Publisher's Note Springer Nature remains neutral with regard to jurisdictional claims in published maps and institutional affiliations. 